\title{
CONDICÕES DE P-T DE FORMACÃO DOS GRANULITOS DO FAROL DA BARRA, SALVADOR, BAHIA, BRASIL
}

\author{
SHIGUEMI FUJIMORI*
}

\begin{abstract}
P-T CONDITIONS OF GRANULITES FORMATION' FROM FAROL DA BARRA, SALVADOR, BAHIA, BRAZIL. The P-T conditions of formation of granulites from Salvador, Bahia, were determined by some available geothermometers and geobarometers. At least, three phases of polimetamorphism were recognized: $7,5 \mathrm{kbar}$ and $840-900^{\circ} \mathrm{C} ; 6,75-7,5 \mathrm{kbar}$ and $525-550^{\circ} \mathrm{C}$; and around $3 \operatorname{kbar} 750^{\circ} \mathrm{C}$.
\end{abstract}

INTRODUCÃo As rochas metamórficas da fácies granulito que aparecem na parte alta da cidade de Salvador foram estudadas petrográfica e mineralogicamente por Fujimori \& Allard (1966) e Fujimori (1968). Algumas variedades foram estudadas quimicamente, como os granulitos com granadas predominantemente almandíferas, do Farol da Barra (Fujimori \& Fyfe 1984), tendo os autores chegado à conclusão de que o protólito original para esse tipo de rocha pode ter sido um solo residual pré-cambriano.

As condições de pressão e temperatura para a formação dessas rochas metamórficas, entretanto, ainda são pouco definidas. Stormer (1973), ao estudar as rochas hipersteníticas com safirina do Rio Vermelho, Salvador, admitiu uma temperatura de cerca de $1.000^{\circ} \mathrm{C}$ para a formação dessas rochas. Stormer \& Whitney (1977), utilizando o equilíbrio entre plagioclásico e feldspato alcalino coexistentes nos granulitos quartzo-feldspáticos dessa região como geotermômetro, concluíram que suas condições de metamorfismo estão na faixa $750^{\circ} \mathrm{C}-800^{\circ} \mathrm{C}$ e $4-8$ kbar.

As condições de pressão e temperatura de formação das rochas metamórficas com granada do Farol da Barra foram delimitadas por alguns geotermômetros e geobarômetros disponíveis: ortopiroxênio-clinopiroxênio (Wood \& Banno 1973), ortopiroxênio-granada (Wood 1974), biotita-granada (Ferry \& Spear 1978), ortopiroxênio-plagioclásio-granada- quartzo (Perkins \& Newton 1981), granada-sillimanita-cordieritaespinélio (Harris 1981) e granada-cordierita (Currie 1971).

PETROGRAFIA As rochas analisadas, expostas no Farol da Barra (Fig. 1), foram descritas com certa minúcia por Fujimori \& Fyfe (1984). As seguintes associações mineralógicas foram estudadas:

Diopsiditos:

a) clinopiroxênio ( \pm plagioclásio \pm quartzo)

Granulitos máficos:

b) quartzo + ortopiroxênio ( \pm plagioclásio)

c) ortopiroxênio + plagioclásio

d) ortopiroxênio + clinopiroxênio + plagioclásio Granulitos félsicos:

e) granada + quartzo \pm plagioclásio (kinzigito)

f) granada + sillimanita + quartzo (khondalito)

g) granada + sillimanita + cordierita + quartzo \pm plagioclásio

Diopsiditos Os diopsiditos são rochas geralmente maciças, de cor esverdeada a esbranquiçada e de granulação fina a grossa. Ocorrem na forma de lentes alinhadas, sugerindo um processo de boudinage. Essas lentes, no Farol da Barra, estão rodeadas por rochas claras, às vezes azuladas, compostas principalmente de quartzo e um pouco de ortopiroxênio ( \pm plagioclásio \pm biotita). Os minerais essenciais dos diopsiditos são diopsídio e quantidades variáveis de plagioclásio, quartzo, biotita, escapolita, epídoto-zoisita. As variedades esbranquiçadas ricas em quartzo podem confundir-se macroscopicamente com os quartzitos. São rochas metamórficas que resultaram de um material sedimentar carbonatado impuro original (Sighinolfi \& Fujimori 1974).

Granulitos maficos São rochas de cor cinza-esverdeada a azulada, granulação fina e sacaroidal. No local estudado, ocorrem duas variedades mineralógicas principais: ortopiroxênio + plagioclásio e ortopiroxênio + clinopiroxênio + plagioclásio (amostras FBG-6 e FBG-7). Além desses minerais essenciais, aparecem em proporções pequenas e variáveis quartzo, hornblenda, biotita, minerais opacos e apatita. Essas associações mineralógicas aparecem em faixas até milimétricas, que se intercalam, conferindo às rochas uma foliação. Os piroxênios foram analisados com microssonda eletrônica (Tab. 1) e como praticamente são coexistentes nessas rochas, às vezes ocorrendo em faixas paralelas numa mesma lâmina delgada, eles foram utilizados para a geotermometria. A composição química dessas rochas (Fujimori \& Fyfe 1984) lançada nos diagramas do tipo De La Roche sugeriu-lhes uma rocha original de natureza mais ígnea intermediária do que sedimentar.

Granulitos félsicos Neste grupo estão as associações granada + quartzo + plagioclásio \pm biotita (kinzigitos) granada + sillimanita + plagioclásio \pm biotita (khondalitos) e granada + sillimanita + cordierita + quartzo + plagioclásio q espinélio \pm coríndon \pm biotita, contendo algumas amostras também ortopiroxênio (FBG-5 e FBG-11). Essas rochas não são distinguíveis macroscopicamente. São de cor cinzaesbranquiçada, granulação fina, sacaroidal e porfiroblásticas. Os porfiroblastos são de granada e, nas rochas com cordierita, esta substitui aquela e é substituída por biotita. Alguns minerais dessas associações possibilitaram a aplicação de geotermômetros e geobarômetros.

Análises químicas dos minerais obtidas pela microssonda eletrônica da Universidade Western Ontário, London, Canadá, permitiram o cálculo, de parâmetros necessários para a determinação da temperatura e pressão, e estão na tabela 2 . $\mathrm{O}$ ferro foi considerado como $\mathrm{FeO}$.

GEOTERMÔMETROS E GEOBARÔMETROS Diversas reações envolvendo o equilíbrio e a substituição foram utilizadas, considerando que nas amostras estudadas esse equilíbrio tenha sido atingido.

Equilíbrio Ortopiroxênio-clinopiroxênio

é baseado na reação:

$$
\underset{\text { (ortopiroxênio) }}{\mathrm{Mg}_{2} \mathrm{Si}_{2} \mathrm{O}_{6}} \underset{\text { (clinopiroxênio) }}{\mathrm{Mg}_{2} \mathrm{Si}_{2} \mathrm{O}_{6}}
$$

Este equilíbrio 


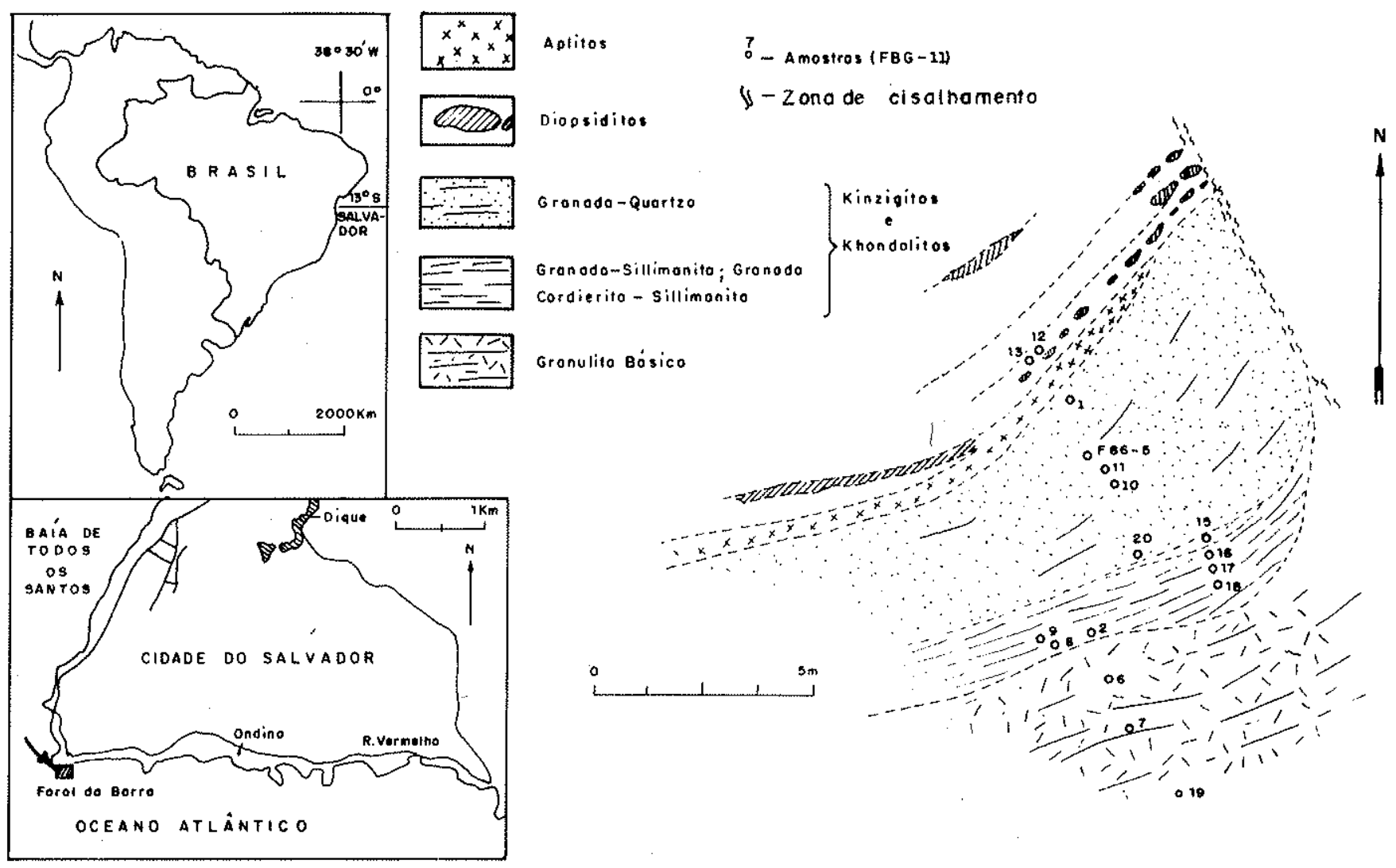

Figura 1-Granulitos granatfferos do Farol da Barra, Salvador, Bahia

A formula utilizada para a determinação da temperatura foi a de Wood \& Banno (op. cit.):

$$
\begin{aligned}
& \mathrm{T}\left({ }^{\circ} \mathrm{K}\right)= \\
& -10.202
\end{aligned}
$$

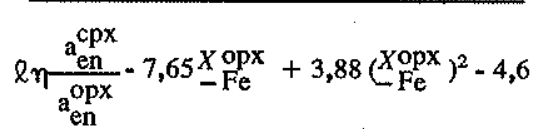

$$
\begin{aligned}
& \underset{-\mathrm{en}}{{ }^{\mathrm{cpx}}}=\left(\underline{X}_{\mathrm{Mg}, \mathrm{M}_{1}}\right)_{\mathrm{cpx}} \cdot\left(\underline{X}_{\mathrm{Mg}, \mathrm{M}_{2}}\right)_{\mathrm{cpx}}= \\
& \left(\frac{\mathrm{Mg}^{2+}}{\mathrm{Mg}^{2+}+\mathrm{Fe}^{2+}+\mathrm{Al}^{3+}}\right)_{\mathrm{M}_{1}}^{\mathrm{cpx}} \cdot\left(\frac{\mathrm{Mg}^{2+}}{\mathrm{Mg}^{2+}+\mathrm{Fe}^{2+}+\mathrm{Ca}^{2+}+\mathrm{Mn}^{2+}}\right)_{\mathrm{M}_{2}}^{\mathrm{cpx}} \\
& \underline{a}_{-\mathrm{en}}^{\mathrm{opx}}=\left(\underline{X}_{\mathrm{Mg}, \mathrm{M}_{1}}\right)_{\mathrm{opx}} \cdot\left(\underline{X}_{\mathrm{Mg}, \mathrm{M}_{2}}\right)_{\mathrm{opx}}= \\
& \left(\frac{\mathrm{Mg}^{2+}}{\mathrm{Mg}^{2+}+\mathrm{Fe}^{2+}+\mathrm{Al}^{3+} \ldots}\right)_{\mathrm{M}_{1}}^{\mathrm{opx}} \cdot\left(\frac{\mathrm{Mg}^{2+}}{\mathrm{Mg}^{2+}+\mathrm{Fe}^{2+}+\mathrm{Ca}^{2+}+\mathrm{Mn}^{2+}}\right) \mathrm{Mpx}_{2}^{\mathrm{opx}} \\
& \text { e } \quad X_{-\mathrm{Fe}}^{\mathrm{opx}}=\left(\frac{\mathrm{Fe}^{2+}}{\mathrm{Fe}^{2+}+\mathrm{Mg}^{2+}}\right) \mathrm{opx}
\end{aligned}
$$

As amostras FBG-6 e FBG-7, muito próximas, forneceram o ortopiroxênio e clinopiroxênio para este cálculo. $\mathrm{O}$ resuitado está na tabela 2 .

Equilibrio ortopiroxênio-granada A granada e o ortopiroxênio coexistentes em equilíbrio nas amostras FBG-5 e FBG-11 podem ser relacionados pela equação:

$$
\begin{aligned}
& \mathrm{Mg}_{2} \mathrm{Si}_{2} \mathrm{O}_{6}+\mathrm{MgAl}_{2} \mathrm{SiO}_{6} \rightarrow \mathrm{Mg}_{3} \mathrm{Al}_{2} \mathrm{Si}_{3} \mathrm{O}_{12} \\
& \text { (ortopiroxênio) (granada) }
\end{aligned}
$$

Para o cálculo de pressão e temperatura de equilíbrio foi utilizada a equação geral $\Delta \mathrm{G}_{\mathrm{P}, \mathrm{T}}=\Delta \mathrm{H}_{298}^{0}-\mathrm{T} . \Delta \mathrm{S}_{298}^{0}+\Delta \mathrm{V}(\mathrm{P}-1)+\mathrm{RT}$ ln $\mathrm{K}=\mathrm{O}(2 \mathrm{a})$ na qual, conforme Wood (op. cit), $\Delta \mathrm{H}^{\circ}=-7.012 \mathrm{cal}^{\mathrm{m}} \mathrm{mol}^{-1} \mathrm{e}$ $\Delta \mathrm{S}_{298}^{\mathrm{o}}=-3,89{\mathrm{cal} . \mathrm{mol}^{-1}}^{-1} \cdot \mathrm{grau}^{-1} . \Delta \mathrm{V}$ foi determinado pela interpolação dos dados de $\left(\mathrm{V}_{\mathrm{Mg}_{3}}^{\circ} \mathrm{Al}_{2} \mathrm{Si}_{3} \mathrm{O}_{12}-\bar{\nabla} \mathrm{Mg}_{2} \mathrm{Si}_{2} \mathrm{O}_{6}-\right.$ $\overline{\mathrm{V}} \mathrm{Mg} \mathrm{Al} \mathrm{SiO}_{6}$ ) em função da porcentagem de $\mathrm{Al}$ na posição $\mathrm{M}_{1}$ do ortopiroxênio, de acordo também com Wood (op. cit.). Por outro lado,

$$
\mathrm{K}=\frac{\frac{a}{\mathrm{Mg}_{3} \mathrm{Al}_{2} \mathrm{Si}_{3} \mathrm{O}_{12}}}{\frac{a_{-}^{\mathrm{opx}} \mathrm{Mg}_{2} \mathrm{Si}_{2} \mathrm{O}_{6} \cdot{ }^{\mathrm{opx}}-\mathrm{MgAl}_{2} \mathrm{SiO}_{6}}{\mathrm{Mg}_{6}}}
$$

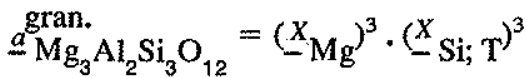

$\stackrel{a}{-\mathrm{Mg}_{2} \mathrm{Si}_{2} \mathrm{O}_{6}}=\left(X_{\mathrm{Mg}} \mathrm{M}_{1}\right)\left(X_{\mathrm{Mg}}, \mathrm{M}_{2}\right) \cdot\left(X_{\mathrm{Si}}, \mathrm{T}\right)^{2}$

$\underline{a}_{\mathrm{MgAl}_{2} \mathrm{SiO}_{6}}^{\mathrm{opx}}=4\left(\underline{X}_{\mathrm{Mg}},{ }_{2}\right)\left(\underline{X} \mathrm{Al}, \mathrm{M}_{1}\right)(\underline{X} \mathrm{Al}, \mathrm{T})(\underline{X} \mathrm{Si}, \mathrm{T})$ (Mukherjee et al. 1968). Os valores de $\mathrm{T}$ e $\mathrm{P}$ calculados para as amostras FBG-5 e FBG-11 estão na tabela 2.

Equilíbrio biotita-granada(substituição) Este equilíbrio de substituição é baseado na seguinte reação:

$$
\begin{gathered}
\underset{3}{\mathrm{Fe}_{3} \mathrm{Al}_{2} \mathrm{Si}_{3} \mathrm{O}_{12}}+\underset{\text { (branada) }}{\mathrm{KMg}_{3}} \mathrm{AlSi}_{3} \mathrm{O}_{10}(\mathrm{OH})_{2} \rightleftarrows \\
\underset{\text { (biotita) }}{\mathrm{Mg}_{3} \mathrm{Al}_{2} \mathrm{Si}_{3} \mathrm{O}_{12}}+\underset{\text { (branada) }}{\mathrm{KFe}_{3} \mathrm{AlSiO}_{3} \mathrm{O}_{10}(\mathrm{OH})_{2}} \\
\text { (biotita) }
\end{gathered}
$$




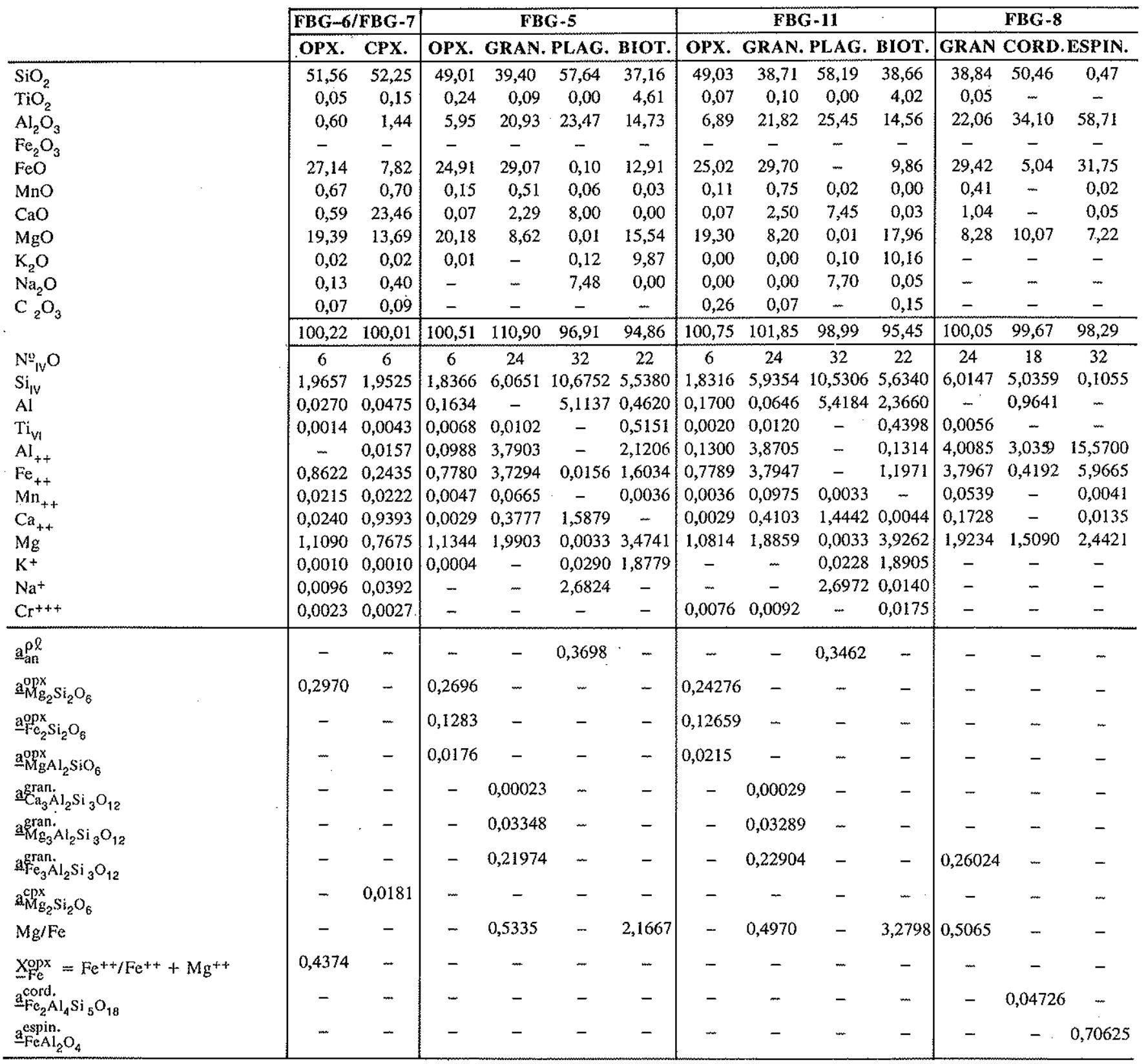

As condiçóes de equilíbrio foram calculadas pela calibração de Ferry \& Spear (op. cit.):

$12.454-4,622 \mathrm{~T}\left({ }^{\circ} \mathrm{K}\right)+0,057 \mathrm{P}(\mathrm{bar})+3 \mathrm{RT} \ln \mathrm{K}=0$, (3a)

na qual $K=\frac{(\mathrm{Mg} / \mathrm{Fe}) \text { granada }}{(\mathrm{Mg} / \mathrm{Fe}) \text { biotida }}$

Os valores calculados de $T$ e $P$ para as amostras FBG-5 e FBG-11, estão na tabela 2.

Equilíbrio Ortopiroxênio - plagioclásio - granada - quartzo É baseado na reação:

$$
\begin{aligned}
& \mathrm{Mg}_{2} \mathrm{Si}_{2} \mathrm{O}_{6}+\mathrm{CaAl}_{2} \mathrm{Si}_{2} \mathrm{O}_{8} \rightarrow \frac{2}{3} \quad \mathrm{Mg}_{3} \mathrm{Al}_{2} \mathrm{Si}_{3} \mathrm{O}_{12} \\
& \text { (enstatita) (anortita) (piropo) }
\end{aligned}
$$$$
+\frac{1}{3} \underset{\text { (glossularita) }}{\mathrm{Ca}_{3} \mathrm{Al}_{2} \mathrm{Si}_{3} \mathrm{O}_{12}}+\underset{\text { (quartzo) }}{\mathrm{SiO}_{2}}
$$

Os valores de $\mathrm{T}$ e $\mathrm{P}$ foram calculados para a amosta FBG-5, utilizando a calibração de Perkins \& Newton (op. cit.):

$\mathrm{P}_{(\text {bar })}=3.944+13,070 \mathrm{~T}\left({ }^{\circ} \mathrm{K}\right)+3,5038 \mathrm{~T}$

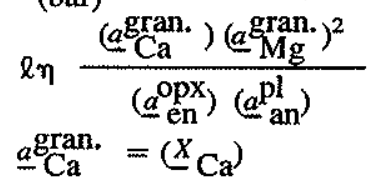

${ }^{a} \stackrel{\operatorname{gran}}{\mathrm{Mg}}=\left(X_{\mathrm{Mg}}\right)$

${ }_{a}^{\mathrm{pl}}=\frac{\mathrm{Ca}}{\mathrm{Ca}+\mathrm{Na}+\mathrm{K}}$

$\underline{a}_{\mathrm{en}}^{\mathrm{opx}}=\left(\underline{\mathrm{Mg}}_{\mathrm{M}, \mathrm{T}}\right)\left(\underline{\mathrm{Xg}}_{\mathrm{Mg}, \mathrm{M}_{2}}\right)\left(\underline{X}_{\mathrm{Si}, \mathrm{T}}\right)^{2}$ 
TABELA 2 - Temperaturas e pressões calculadas as

\begin{tabular}{|c|c|c|c|c|c|c|}
\hline \multirow{2}{*}{$\frac{\text { R E A Ç Ã O }}{\text { [1] opx-cpx (Wood \& Branno 1973) }}$} & \multirow{2}{*}{$\begin{array}{c}\text { AMOSTRA } \\
\text { FBG-6 x FBG-7 }\end{array}$} & \multicolumn{5}{|c|}{ TEMPERATURA/PRESSÃO } \\
\hline & & $\mathrm{T}^{\circ} \mathrm{C}$ & 747 & & & \\
\hline [2] opx-granada (equilíbrio) (Wood 1974) & $\begin{array}{l}\text { FBG-5 } \\
\text { FBG-11 }\end{array}$ & $\begin{array}{l}\mathrm{P}(\mathrm{kbar}) \\
\mathrm{T}^{\circ} \mathrm{C} \\
\mathrm{T}^{\circ} \mathrm{C}\end{array}$ & $\begin{array}{c}3 \\
708 \\
737 \\
\end{array}$ & $\begin{array}{c}5 \\
760 \\
791 \\
\end{array}$ & $\begin{array}{c}8 \\
838 \\
873\end{array}$ & $\begin{array}{l}10 \\
890 \\
927\end{array}$ \\
\hline [3] bio-granada (substituição) (Ferry \& Spear 1978) & $\begin{array}{l}\text { FBG-5 } \\
\text { FBG-11 }\end{array}$ & $\begin{array}{l}\mathrm{P}(\mathrm{kbar}) \\
\mathrm{T}^{\circ} \mathrm{C} \\
\mathrm{T}^{\circ} \mathrm{C}\end{array}$ & $\begin{array}{c}3 \\
543 \\
522\end{array}$ & $\begin{array}{c}5 \\
550 \\
529\end{array}$ & $\begin{array}{c}8 \\
562 \\
540\end{array}$ & $\begin{array}{l}10 \\
569 \\
547\end{array}$ \\
\hline [4] opx-plag-gran-qzo (Perkins \& Newton 1982) & $\begin{array}{l}\text { FBG-11 } \\
\text { FBG-5 }\end{array}$ & $\begin{array}{l}\mathrm{T}^{\circ} \mathrm{C} \\
\mathrm{P} \text { (kbar) } \\
\mathrm{P} \text { (kbar) }\end{array}$ & $\begin{array}{l}500 \\
7,3 \\
6,6\end{array}$ & $\begin{array}{l}700 \\
8,2 \\
7,3\end{array}$ & $\begin{array}{l}900 \\
9,0 \\
8,0\end{array}$ & \\
\hline [5] gran.-sill.-cord.-espin. (Harris 1981) & FBG-8 & $\begin{array}{l}\mathrm{T}^{\circ} \mathrm{C} \\
\mathrm{P} \text { (kbar) }\end{array}$ & $\begin{array}{l}500 \\
4,5\end{array}$ & $\begin{array}{l}700 \\
5,3\end{array}$ & $\begin{array}{l}900 \\
6,0\end{array}$ & \\
\hline [6] gran.-cord. (Currie 1971) & FBG-8 & $\begin{array}{l}\mathrm{T}^{\circ} \mathrm{C} \\
\mathrm{P} \text { (kbar) }\end{array}$ & $\begin{array}{l}751 \\
2,9\end{array}$ & & & \\
\hline
\end{tabular}

opx $=$ ortopiroxênio $; \mathrm{cpx}=$ clinopiroxênio; bio = biotita; $\mathrm{plag}=$ plagiocásio; gran. $=$ granada $;$ qzo = quartzo; sill. $=$ sillimanita; cord. $=$ cordierita e espin $=$ espinélio.

Os valores calculados de T e P estão na tabela 2.

Equilíbrio granada-Sillimanita-cordierita-espinélio reação de equilíbrio estudada por Harris (op. cit.) é a seguinte: granada + sillimanita $\rightarrow$ cordierita + espinélio (5)

Para esta reação, obteve-se a equação:

$$
\mathrm{P}_{(\text {bar })}=1+\frac{1.358+(\ln \mathrm{K}+0,82) \mathrm{T}\left({ }^{\circ} \mathrm{K}\right)}{0,7908} \text { na qual }(5 \mathrm{a})
$$

$\mathrm{K}=\frac{\left(\underline{a}_{\mathrm{Fe}} \mathrm{Al}_{2} \mathrm{Si}_{3} \mathrm{O}_{12}\right)}{\left(\underline{a} \mathrm{Fe}_{2} \mathrm{Al}_{4} \mathrm{Si}_{5} \mathrm{O}_{18}\right)\left(\underline{a}_{-}^{\text {corpin. }} \mathrm{Fe}_{2} \mathrm{O}_{4}\right)} \quad$ sendo

$\stackrel{\stackrel{a}{\text { gran. }}{ }_{-} \mathrm{Fe}_{3} \mathrm{Al}_{2} \mathrm{Si}_{3} \mathrm{O}_{12}}{=}\left(\underline{X}_{\mathrm{Ke}}^{\text {gran. }}\right)^{3} ; \underline{a}_{-\mathrm{Fe}_{2} \mathrm{Al}_{4} \mathrm{Si}_{5} \mathrm{O}_{18}}^{\text {cord. }}=\left(\underline{X}_{\mathrm{Fe}}^{\text {cord. }}\right)^{2} \mathrm{e}$

$\underline{\mathrm{FeAl}}_{2} \mathrm{O}_{4}=\left(\underline{X}_{\mathrm{Fe}}^{\text {espin. }}\right)\left(\underline{X}_{\mathrm{Al}}^{\text {espin. }}\right)$

Os valores de $\mathrm{T}$ e $\mathrm{P}$ calculados por essa fórmula para a amostra FGB-8 estão na tabela 2.

Equilíbrio Cordierita-Granada Baseando-se na cordierita e granada, Currie (op. cit) apresentou um geotermômetro e geobarômetro. As fórmulas para temperatura e pressão são:

$$
\begin{aligned}
& \mathrm{T}=\frac{4.515}{6,37-\operatorname{l\eta } \mathrm{K}_{\mathrm{D}}},(6) \text { em que } \mathrm{K}_{\mathrm{D}}=\frac{(\mathrm{Mg} / \mathrm{Fe}) \text { cord. }}{(\mathrm{Mg} / \mathrm{Fe}) \text { gran. }} \mathrm{e} \\
& \left.\mathrm{P}=\mathrm{P}^{\circ}+\frac{\Delta \mathrm{P}^{\circ}}{2} \underline{X}_{\text {gran. }}+\underline{X}_{\text {cord. }}\right) \quad(6 \mathrm{a}), \text { em que } \\
& \underline{X}=\frac{\mathrm{Mg}}{\mathrm{Mg}+\mathrm{Fe}} \text { e } \Delta \mathrm{P}^{\circ}=0,003138 \mathrm{~T} \ln \mathrm{K}_{\mathrm{D}}
\end{aligned}
$$

$\mathrm{P}^{\circ}=$ pressão de quilíbrio da granada $\mathrm{Fe}$ e cordierita $\mathrm{Fe}$, na temperatura calculada T, da reação: $\frac{1}{2}$ cordierita $\mathrm{Fe} \rightleftarrows$ $\frac{1}{3}$ granada $\mathrm{Fe}+\frac{2}{3}$ sillimanita $+\frac{5}{6}$ quartzo e para a qual

$$
\begin{aligned}
& \Delta H^{\circ}=+38.250 \mathrm{cal} \\
& \Delta \mathrm{S}^{\circ}=26,20 \mathrm{U} . \mathrm{E} . \\
& \Delta \mathrm{V}^{\circ}=-164.2 \mathrm{~cm}^{3} \\
& \mathrm{O} \text { valor de } \mathrm{P}^{\circ} \text { e obtido pela expressão geral } \\
& \Delta \mathrm{G}_{\mathrm{P}, \mathrm{T}}=\Delta \mathrm{H}^{\circ}-\mathrm{T} \Delta \mathrm{S}^{\circ}+\left(\mathrm{P}^{\circ}-1\right) \Delta \mathrm{V}=\mathrm{O}
\end{aligned}
$$

Os valores de $\mathrm{T}$ e $\mathrm{P}$ calculados para a amostra FBG- 8 estão na tabela 2.

Os dados geotermométricos e geobarométricos obtidos pelas diversas fórmulas estão representados graficamente no diagrama P-T (fig.2), ao qual foram acrescentadas as retas de equilíbrio para os polimorfos de $\mathrm{Al}_{2} \mathrm{SiO}_{5}$ (cianitaandaluzita-sillimanita) calculadas pelos dados termodinâmicos fornecidos para esses componentes por Robie \& Waldbaum (1968).

CONCLUSÕES As condições metamórficas de formação dos granulitos de Salvador foram delimitadas por alguns geotermômetros e geobarômetros calibrados, com introdução de simplificações em seus cálculos. O princípio da convergência de equilíbrio (Mukherjee et al. op. cit.) especifica que as interseções das curvas de geotermômetros e geobarômetros para uma mesma associação mineral determinam as condições de $\mathrm{P}$ e de $\mathrm{T}$ para esse conjunto. A precisão desses sensores $\varepsilon$ difícil de ser estabelecida, pois depende da calibração dos geotermômetros e geobarômetros. Apesar dessas limitaçőes, entretanto, as reaçőes 2,3 e 4 indicam (Fig. 2) duas condições de metamorfismo em torno de $6,75-7,5$ kbar e $525-550^{\circ} \mathrm{C}$ (B) e 7,5-9 kbar e $840-900^{\circ} \mathrm{C}$ (A). $\mathrm{O}$ geotermômetro e o geobarômetro de Currie (op. cit.) e as reaçōes 5 e 6 evolvendo a cordierita indicam uma outra condição em torno de $750^{\circ} \mathrm{C}$ e $3 \mathrm{kbar}$ (C) distinta das duas primeiras.

Essas três condições podem representar as diversas fases do polimetamorfismo sofrido pelos granulitos de Salvador. Após atingir o pico do metamorfismo (A), sob condições de 


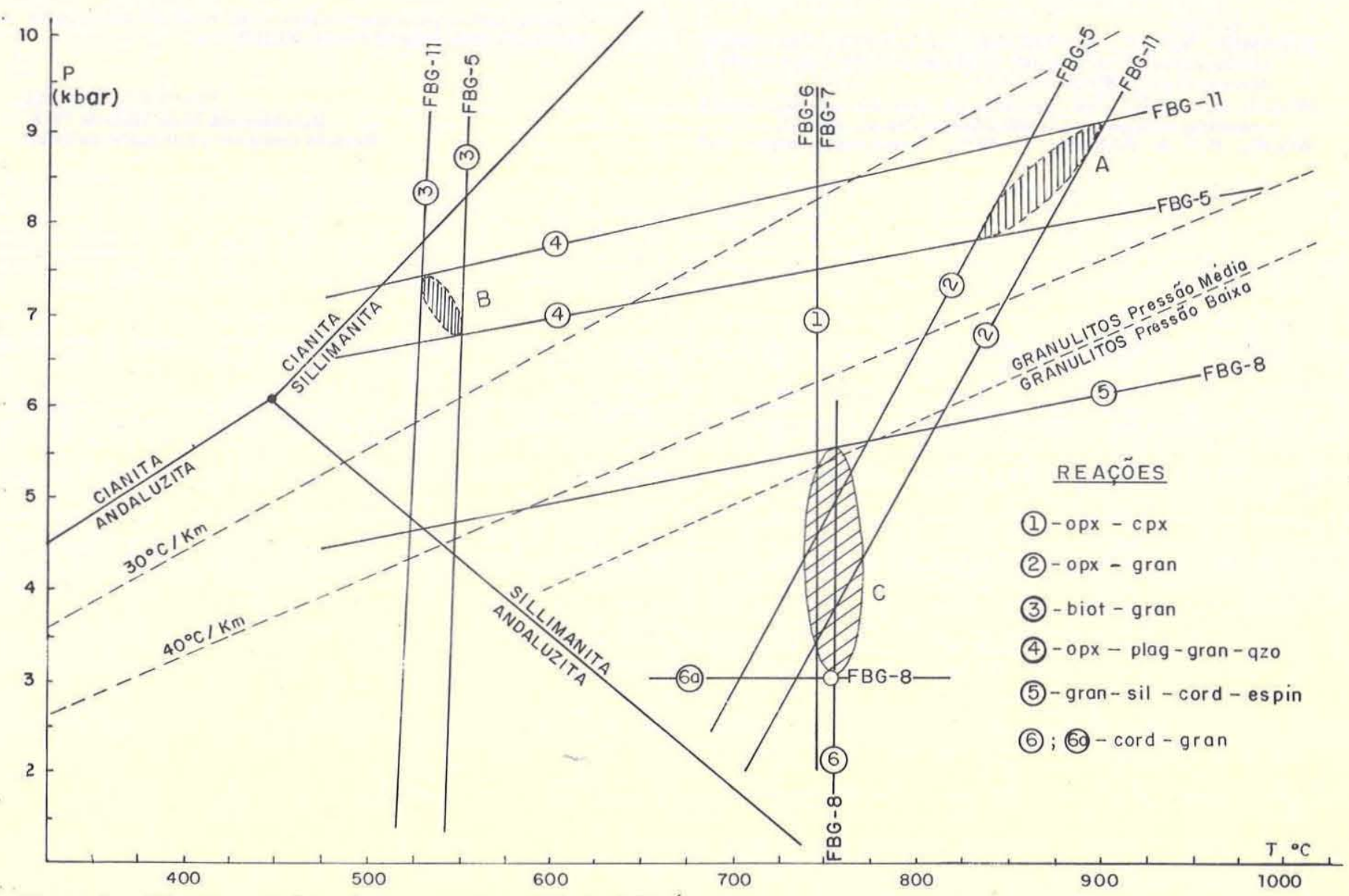

Figura 2 - Diagrama sinótico das curvas de equilfbrio P-T. Áreas hachuradas = convergências de P-T para granulitos de Salvador, Bahia. Reta que separa granulitos de baixa e média pressões de acordo com Green \& Ringwoord (1967)

fácies granulito de pressão intermediária (Green \& Ringwood 1967), houve um retrometamorfismo, principalmente com o abaixamento da pressão, dando origem à associação com cordierita (C), e posteriormente um outro episódio para dar origem à associação com biotita (B).

Os geotermômetros e geobarômetros se baseiam em paragêneses nas diversas condiçōes de $T$ e $P$ e as transformações polimetamórficas constatadas são função também da composição química das rochas, permitindo a preservação das associações mineralógicas anteriores. A paragênese com biotita possivelmente resultou de um processo de cisalhamento sofrido pelas rochas.

Os valores obtidos por Stormer (op. cit.) e Stormer \& Whitney (op. cit.) estão também nessas faixas. A temperatura de $1.000^{\circ} \mathrm{C}$ obtida para rochas com hiperstênio e safirina, situadas a cerca de $5 \mathrm{~km}$ a leste do Farol da Barra, poderá corresponder às paragêneses de alta temperatura.

Agradecimentos Os agradecimentos são devidos ao Conselho Nacional de Desenvolvimento Científico e Tecnológico $(\mathrm{CNPq})$, pela bolsa de pesquisa proporcionada, e à Sra. Eraldina Leão Campelo e ao Sr. Warney Bispo de Magalhães, pela datilografia e confecção de desenhos, respectivamente. As análises químicas dos minerais utilizando a microssonda eletrônica foram possíveis graças à Canadian Internacional Development Agency (CIDA), que proporcionou um estágio no Departamento de Geologia da Universidade de Western Ontário, London, Canadá.

\section{REFERÊNCIAS BIBLIOGRÁFICAS}

CURRIE, K.L. 1971. The reaction 3 cordierite $=2$ garnet +4 sillimanite +5 quartz as a geological thermomether in the Opinion Lake Region, Ontario, Contr. Mineral. Petrol., 33:215-226

FERRY; J.M. \& SPEAR F.S. 1978. Experimental calibration of the partioning of $\mathrm{Fe}$ and $\mathrm{Mg}$ between biotite and garnet. Contrib. Mineral. Petrol., 66:113-117.

FUЛМОRI, S, 1968. Granulitos e charnockitos de Salvador, Bahia. An.Acad, Brasil. Ciênc, 40:181-202.

FUJIMORI, S. \& ALLARD, G.O. 1966. Ocorrência de safirina em Salvador, Bahia. Bol. Soc. Bras. Geol., 15:67-81

FUJIMORI, S. \& FYFE, W.S. 1984. Almanditic garnet-rich metamorphic rocks as an original soil developed during Precambian, Rev, Bras, Geoc, 14(4):194-202.

GREEN, D.H. \& RINGWOOD, A.E. 1967. An experimental, investigation of the gabbro to eclogite transformation and its petrological applications. Geochim. Cosmoch. Acta, 31:767-833.

HARRIS, N, 1981. The application of spinel-bearing metapelites to P/T determinations: an exemple from South India. Contrib. Mineral.
Petrol., 76:229-233.

MUKHERJEE, A.; BHATTACHARYA, A.; CHARKRABORTY, S.C. 1986. Convergent phase equilibra at the massif anorthosit-granulite interface near Bolangis, Orissa, India, and thermal evolution of a part of the India Shield. Precambrian. Res., 34:69-104.

PERKINS III, D. \& NEWTON, R.C. 1981. Charnockite geobarometers based on coexisting garnet-pyroxene-plagioclase-quartz. Nature, 292:144-146.

ROBIE, R.A. WALDBAUM, D.R. 1968. Thermodynamic properties of minerals and related substances at $298,15^{\circ} \mathrm{K}\left(25,0^{\circ} \mathrm{C}\right)$ and one atmosphere (1,0138 bars) pressure at higher temperatures. US. Geology. Surv, 1259, $255 \mathrm{pp}$

SIGHINOLFI, G.P. \& FUЛMORI, S. 1974. Petrology and chemistry of diopsidic rocks in granulite terrains from the Brazilian basement. Atti della Soc. Tosc. Di Sci. Natur., serie A, LXXXI: $103-120$.

STORMER Jr., J.C. 1973. The composition of sapphirine from Salvador, Bahia, and conditions of its formation, Rev. Bras. Geoc., 
3:192-200.

STORMER Jr., J.C. \& WHITNEY, J.A. 1977. Two-feldspar geothermometry in granulite facies metamorphic rocks. Contrib. Mineral.Petrol., 65:123-133.

WOOD, B.J. 1974. The solubility of alumina in orthopyroxene coexisting with garnet. Contrib. Mineral. Petrol., 46:1-15.

WOOD, B.J. \& BANNO, S. 1973. Garnet-ortopyroxene and orthopyroxene-clynopiroxene relationships in simple and complex systems. Contrib. Mineral. Petrol., 42:109-124.

MANUSCRITO: 464

Recebido em 22 de Maio de 1988. Revisão aceita em 14 de junho de 1988.

"Defrontei-me, de repente, com uma geografia do mal acabado, de uma violência desconcertante. A linha constante de água e mato servia de moldura a um mundo ainda ingógnito e confuso. As florestas não tinham fim. A terra se repetia, carregada de alaridos anônimos. Eram vozes indecifradas. Sempre água e mato por toda a parte, aquele sem-fim de mato, de um verde imenso, com árvores de mãos dadas, ao londo da margem do grande rio". 